\title{
Chlamydia pneumoniae Accelerates Coronary Artery Disease Progression in Transgenic Hyperlipidemia-Genetic Hypertension Rat Model
}

\author{
Victoria LM Herrera, ${ }^{1}$ Li Shen, $^{2}$ Lyle V Lopez, $^{1}$ TAMARA DidishVili, $^{1}$ You-Xun Zhang, ${ }^{2}$ \\ AND NELSON RUIZ-OPAZO ${ }^{1}$
}

Chlamydia pneumoniae ( $\mathrm{Cpn}$ ) has been associated with human coronary artery disease but causal relevance as a risk factor has not been shown. Several rabbit and mouse model studies demonstrate exacerbation of aortic atherosclerosis by Cpn, however impact of Cpn on coronary artery disease (CAD) and survival outcomes has not been shown. To study this, we used specific pathogen-free, inbred, transgenic-CAD Dahl salt-sensitive (S) hypertensive (Tg53) rats and control inbred, non-transgenic Dahl S (nonTg) rats to analyze the effects of Cpn infection on macrophage foam cell formation, coronary artery disease progression, and effect on survival. Cpn infection induced acceleration of foam cell formation in hyperlipidemic Tg53 recruited peritoneal macrophages. This effect is hyperlipidemia-dependent. The transcription profile of Tg53-Cpn macrophage foam cells is different from control mock-inoculated (Tg53-spg) and heat-inactivated (Tg53-iCpn) macrophages (ANOVA $P<0.0001$ ). Decreased survival was detected in Tg53-Cpn compared with control nonTg-Cpn and mock-infected Tg53-mouse pneumonitic rats $(P=0.009)$ and was associated with "culprit" coronary plaques and left atrial thrombi. These data demonstrate that in the presence of significant hyperlipidemia and hypertension, one-time Cpn infection at 5 mo of age (associated with early CAD stage) accelerates progression to overt-CAD in the Tg53 rat model. The data support the hypothesis that untreated Cpn infection is a causal risk factor for CAD progression most likely mediated by Cpninduced accelerated macrophage foam cell formation.

\section{INTRODUCTION}

Coronary heart disease remains a leading cause of morbidity and mortality. The delineation of pathogenic mechanisms involved in the evolution of the coronary atherosclerotic plaque from a quiescent to a destabilized plaque underlying overt coronary artery disease is critical. The central role of inflammation in coronary plaque development and destabilization (1) prompts the investigation of infectious disease pathways in plaque progression. Chlamydia pneumoniae (Cpn), a common human respiratory pathogen has been linked to coronary heart disease by seroepidemiological studies $(2,3)$, detection of Cpn in the vascular system (4), and by isolation of Cpn from coronary lesions (5), indicating association short of causality. A complex interaction is apparent. Clinical studies testing whether antibiotic treatment alters the course of coronary artery disease (CAD) present conflicting observations of benefit (6) and non-benefit (7). Different animal model studies have demonstrated multiple effects of Cpn on aortic atherosclerosis pathogenesis, but none have shown the effects on CAD specifically. Observations show that Cpn infection affects the development of aortic atherosclerosis in rabbits (8), aortic root lesion initiation in diet-induced C57/BL6 atherosclerosis mouse model (9), aortic root lesion extent in LDL receptor-deficient (10) and apolipoprotein E-deficient mice $(11,12)$, and aortic arch lesion complexity in apolipoprotein E3-Leiden mice (13). However, some studies show no effects from $\mathrm{Cpn}$ on aortic root atherosclerosis in apolipoprotein E-deficient mice $(14,15)$. Studies also show that the effect is specific to Cpn since infection with Chlamydial mouse pneumonitis (MoPn) strain does not exacerbate aortic atherosclerosis $(10,14)$.

In order to address the role of Cpn in coronary plaque progression and destabilization, we investigated the effects of a onetime Cpn infection in a rat model of coronary artery disease, Tg53 rat model transgenic for human cholesteryl ester transfer protein. Our data demonstrate that Cpn infection accelerates macrophage foam cell formation in vivo in recruited peritoneal macrophages and coronary plaque progression leading to overt coronary artery disease in Tg53 male rats. Transcription profiling of Cpn-induced accelerated macrophage foam cells provide insight into hypotheses for further study.

\section{MATERIALS AND METHODS}

Transgenic Rat Model of Coronary Atherosclerosis We used an inbred transgenic rat model of coronary atherosclerosis with hypertension and hyperlipidemia as risk factors, Tg53 rat model. This model is transgenic for the human cholesteryl ester transfer protein, developed and maintained in inbred Dahl 
salt-sensitive (S) hypertensive strain (17). Combined hyperlipidemia in this model is characterized by low HDL, high total cholesterol, high triglyceride on regular rat chow (17). All test and control rats are inbred Dahl S strain, thus eliminating genetic background as confounder of the atherosclerosis phenotype. Transgenic and control rats were weaned at $4 \mathrm{wk}$ and placed on a regular rat chow diet $(0.4 \% \mathrm{NaCl})$. Lipid profiles were determined as described (17). Animal manipulations complied with institutional care and use protocols. A total of 40 male rats were used for analyses.

\section{Culture, Inoculation, and Documentation of $C p n$ Infection}

Chlamydia pneumoniae (strain AR-39) was grown in HEp-2 cells (Washington Research Foundation, Seattle, WA, USA) and purified by density gradient centrifugation using Renographin 60 (Bracco Diagnostics, Princeton, NJ). The purified organisms were resuspended in sucrose phosphate glutamic acid chlamydial transport medium (spg, $\mathrm{pH} 7.4$ ) and frozen at $-80{ }^{\circ} \mathrm{C}$ until use. Intranasal inoculations were done under light anesthesia. Cpn was given intranasally in $50 \mu \mathrm{L}$ aliquots containing $1 \times 10^{7}$ inclusion forming units (ifu). Mock infection was done with vehicle alone (spg) or with a mouse strain of Chlamydia trachomatis, which causes mouse pneumonitis (MoPn at $1 \times 10^{4} \mathrm{ifu}$ ) following identical procedure. These amounts were shown previously to be nonlethal in LDL receptor (LDLr) null mutant mice (10). Intraperitoneal infection was done by injecting $1 \times 10^{7}$ ifu of Cpn in $100 \mu \mathrm{L}$ vehicle compared with $100 \mu \mathrm{L}$ of heat-inactivated Cpn (iCpn) (identical titer) or vehicle alone. Heat inactivation was done at $65^{\circ} \mathrm{C}$ for $30 \mathrm{~min}$. Cpn infection was tested by culture on HEp2 cell monolayers and immunostaining with a monoclonal antibody C1A6 (unpublished data) against chlamydial lipopolysaccharide, as well as by PCR amplification of Cpn S16 ribosomal DNA from rats injected with $\mathrm{Cpn}$ intraperitoneally in contrast to mock-infected rats. Amplification primers used were: 5' ACG-TCA-CGT-AGT-TAT-AGA-TAAGAG $3^{\prime}$ and 5' AAG-TAG-CTG-GAG-AGG-TAT-CCA-CGG 3'. MoPn was cultured, isolated, and purified in HEp-2 cells essentially as described (10). Inoculation was done following identical procedures for Cpn described above.

\section{Peritoneal Macrophage Foam Cell Analysis}

Recruitment of peritoneal macrophages was done by injecting 3\% thioglycolate intraperitoneally. After $3 \mathrm{~d}$, intraperitoneal injections of Cpn or vehicle (mock-infection) were done under anesthesia into transgenic Tg53 rats and control nontransgenic Dahl S rats. Peritoneal macrophages were collected in Hank's buffer under sterile conditions and analyzed after $1 \mathrm{wk}$ and $2 \mathrm{wk}$ postinfection or post-mock-infection. Tissues were collected under sterile conditions for culture or PCR analysis of Cpn-specific DNA sequences. Cell smears were fixed in phosphate buffered saline (PBS)-buffered 2\% paraformaldehyde solution for $15 \mathrm{~min}$, air dried then kept at $-70{ }^{\circ} \mathrm{C}$. $1 \%$ Oil-red-O (in $60 \%$ isopropanol) staining was done for 15 min essentially as described (18).

\section{Transcription Profiling}

Total cellular RNA was isolated from frozen peritoneal macrophage cell pellet after PBS wash. RNA was checked for quality and amount by spectrophotometry and RNA gel analysis. Transcription profiling was done with the Mergen RO-1 rat microarray specific for rat 3' untranslated sequences as per manufacturer's specifications (Mergen Ltd, San Leandro, CA, USA). Each microarray has 1046 unique gene sequences with multiple internal positive and negative controls. Analysis was done following stringent criteria essentially as described (19). Specific to the study's data set, 2-fold change was determined to be $>2$ standard deviations from the mean of $\log _{2}$ transformed expression ratios for expression levels $>100$. Statistical analysis was done using ANOVA and Tukey's multipoint comparison (PRISM, Graphpad Software, San Diego, CA, USA).

\section{Immunohistochemical Analysis}

Immunohistochemical analysis was done as described (17). Antibodies used were anti-monocyte/macrophage subset antibody (Pharmingen, San Diego, CA, USA), and chlamydial anti-Hsp60 antibody (Chemicon International Inc, Temecula, CA, USA) and anti-MMP3 (stromelysin) antibody (Santa Cruz Biotechnology, Santa Cruz, CA, USA).

\section{Survival Analysis Measured as Time to Onset of Overt CAD}

At 5 mo of age, transgenic Tg53 male rats were inoculated intranasally with Cpn (Tg53-Cpn, $n=4)$. Controls $(n=8)$ were comprised of 2 subgroups: transgenic Tg53 male rats inoculated with MoPn (Tg53-MoPn, $n=5$ ) and nontransgenic Dahl S rats inoculated with Cpn (nonTg-Cpn, $n=3$ ). They were housed 2 per cage and observed for any distress. All inoculations were tolerated well with no distress in the immediate 2 -wk period, and there were no premature deaths in either control group. Having determined no premature deaths due to Cpn-infection, and following institutional animal care policies, life span analysis was done using onset of empirical distress to prompt euthanasia as experimental end point determined by animal technicians in a blinded manner. Hearts were processed for histopathology. This experimental end point was previously shown to be associated with "culprit" coronary plaques in Tg53 rats, which have never been detected in nontransgenic Dahl S controls (17). Statistical analysis of group means was done using $t$ test (PRISM).

\section{Histopathology Analysis of Coronary Artery Disease}

Hearts were collected, rinsed in cold PBS, fixed in cold PBSbuffered $4 \%$ paraformaldehyde, embedded in paraffin, and sectioned at 6 microns. Serial sections (300) were stained with Masson Trichrome every 15 sections and assessed for lesions essentially as described (17). Adjacent sections were used for immunohistochemical analysis or phosphotungstic acid hematoxylin (PTAH) staining to detect fibrin-positive thrombi.

\section{RESULTS}

\section{Cpn Accelerates Macrophage Foam Cell Formation}

Because macrophage foam cells are associated with coronary plaque progression and vulnerable plaque destabilization, we first determined whether Cpn infection would accelerate macrophage foam cell formation in an in vivo experimental sys- 
Table 1. Analysis of foam cell formation in recruited peritoneal macrophages ${ }^{a}$

Time elapsed from inoculation

\begin{tabular}{|c|c|c|c|c|c|c|c|}
\hline \multirow[b]{2}{*}{ Study groups } & \multicolumn{2}{|c|}{$1 \mathrm{mo}$} & \multicolumn{2}{|c|}{$2 w k$} & \multicolumn{3}{|c|}{$1 \mathrm{wk}$} \\
\hline & Cpn & spg & Cpn & spg & Cpn & spg & iCpn \\
\hline $\operatorname{Tg} 53(n=20)$ & $1^{b}$ & ND & $1^{b}$ & 1 & $8\left(5^{b} ; 3^{c}\right)$ & $6\left(4^{\mathrm{b}} ; 2^{\mathrm{c}}\right)$ & $+3^{c}$ \\
\hline Foam cell formationd & - & NA & +++ & + & ++++ & - & + \\
\hline Dahl S nonTg $(n=10)$ & 1 & ND & 1 & 1 & $4^{b}$ & 3 & ND \\
\hline Foam cell formation & - & NA & - & - & - & - & NA \\
\hline
\end{tabular}

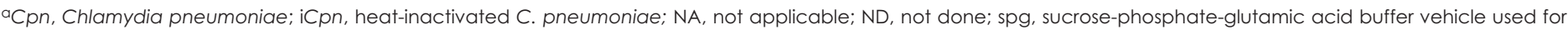

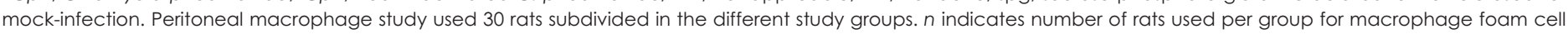
analysis. Some groups are further subdivided as follows.

bRats used for DNA isolation for Cpn ribosomal 16 DNA sequence detection by PCR amplification.

cRats used for macrophage total cellular RNA isolation for transcription profiling.

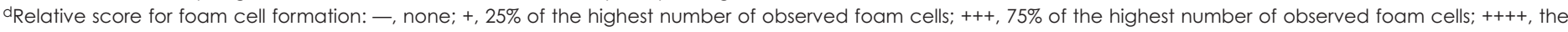
highest number of observed foam cells.

tem simulating observations in cultured human monocytes (20). In order to have an in vivo experimental system combining hyperlipidemia and an easily accessible macrophage pool, we used recruited peritoneal macrophages in hyperlipidemic Tg53 Dahl S rats as experimental system. Intraperitoneal inoculation of Cpn or vehicle was done $3 \mathrm{~d}$ after recruitment of peritoneal macrophages in Tg53 $(n=10)$ and control nontransgenic, nonhyperlipidemic Dahl S rats $(n=6)$ (Table 1$)$. As control, vehicle was inoculated into $\operatorname{Tg} 53(n=7)$ and nontransgenic Dahl S $(n=4)$ rats (Table 1). To confirm Cpn infection, PCR amplification of Cpn-specific DNA was done testing 11 Cpn-inoculated rats (7 Tg53 and 4 nonTg rats) and 4 vehicle-inoculated Tg53 rats (see Table 1). PCR amplification detected Cpn-specific DNA sequences in Cpninoculated rats - both transgenic and control nontransgenic Dahl $S$ rats. C $p n$-specific DNA sequences were amplified from DNA from the following tissues: macrophages $=$ lung $>$ spleen $>$ liver $>$ heart (data not shown). As would be expected, no Cpn-specific DNA sequences were detected in control mock-inoculated rats (data not shown). Cpn-infection did not affect lipid levels (data not shown) as previously observed (10).

In order to assess impact of Cpn-infection on macrophage foam cell formation, oil-red-O staining of cell smears was done to demonstrate characteristic foam cell lipid accumulation (Figure 1). Cell smears of recruited peritoneal macrophages from Tg53-Cpn (see Figure 1A), control mock-infected Tg53-spg (see Figure 1B), and control nonTg-Cpn (see Figure 1C) rats exhibited similar macrophage composition as determined by immunohistochemical staining with a macrophage subset antibody (see Figure 1A through 1C). This validates comparative analysis of cell smears. One week after Cpn- or mock-inoculation, oil-red-O stained lipidladen macrophage foam cells were detected only in Tg53-Cpn peritoneal macrophages (see Figure 1D) and not in either control group, hyperlipidemic Tg53-spg (see Figure 1E) or normolipidemic nonTg-Cpn rats (see Figure $1 \mathrm{~F}$ ). At 2 wk post-inoculation, oil-red-O staining detected few foam cells in mock-inoculated Tg53-spg macrophages but significantly much less compared with Tg53-Cpn macrophages. We note however, that the total numbers of peritoneal macrophages was decreased in both groups (data not shown) indicating a suboptimal experimental time point. At $4 \mathrm{wk}$, minimal peritoneal macrophages were detected in all rats, indicating the experimental nonfeasibility of this time point (data not shown). High magnification corroborates oil-red-O-stained lipidladen macrophage foam cells in Tg53-Cpn (see Figure 1G). In order to show equivalent C $p n$ infection, immunohistochemical analysis with anti-HSP60 Cpn antibody detects immunostained macrophages in both Cpn-infected Tg53-Cpn (see Figure 1H) and nonTgCpn rat groups (see Figure 1I).

Collectively, the data demonstrate that only recruited peritoneal macrophages from hyperlipidemic Tg53 rats develop foam cells; that Cpn-infection accelerates the formation of foam cells affecting both onset and number; and that Cpn-infection without hyperlipidemia is not sufficient to induce macrophage foam cell formation.

\section{Transcription Profiling of Recruited Peritoneal Macrophage Foam Cells}

In order to gain insight into transcriptional events underlying Cpninduced acceleration of macrophage foam cell formation detected at 1 wk post-inoculation, we determined the macrophage transcription profiles comparing Tg53-Cpn rats (pooled, $n=3$ ), heat-inactivated Cpn-inoculated rats, Tg53-iCpn (pooled, $n=3$ ), and control vehicle-inoculated Tg53-spg rats (pooled, $n=2$ ) following a loop design and fulfilling biological replication (21). Cell smear analysis corroborated marked foam cell formation in Tg53-Cpn, minimal in Tg53-iCpn, and none in Tg53-spg recruited peritoneal macrophages.

Comparative scatter plot analyses of transcription profiles (Figure 2) of Tg53-Cpn, Tg53-spg, and Tg53-iCpn demonstrate that the majority of genes on the microarray are expressed in recruited peritoneal macrophages (in blue) in contrast to those genes not expressed (in green) (see Figure 2A through 2C). Only a small subset of genes exhibit $\geq 2$-fold change in macrophage foam cells in Tg53-Cpn rats compared with both controls, macrophages from Tg53-spg and Tg53-iCpn rats (see Figure 2A and 2B respectively). One-way ANOVA of $\log _{2}$ transformed expression ratios (22) from the 3 study groups detected significant differences $(P<0.0001)$. Multipoint comparison analysis reveals that expressed gene profile of Tg53-Cpn is significantly different from Tg53-iCpn $(P<$ $0.001)$ and from Tg53-spg $(P<0.001)$, and that expressed gene profile Tg53-iCpn is not different from Tg53-spg control. 


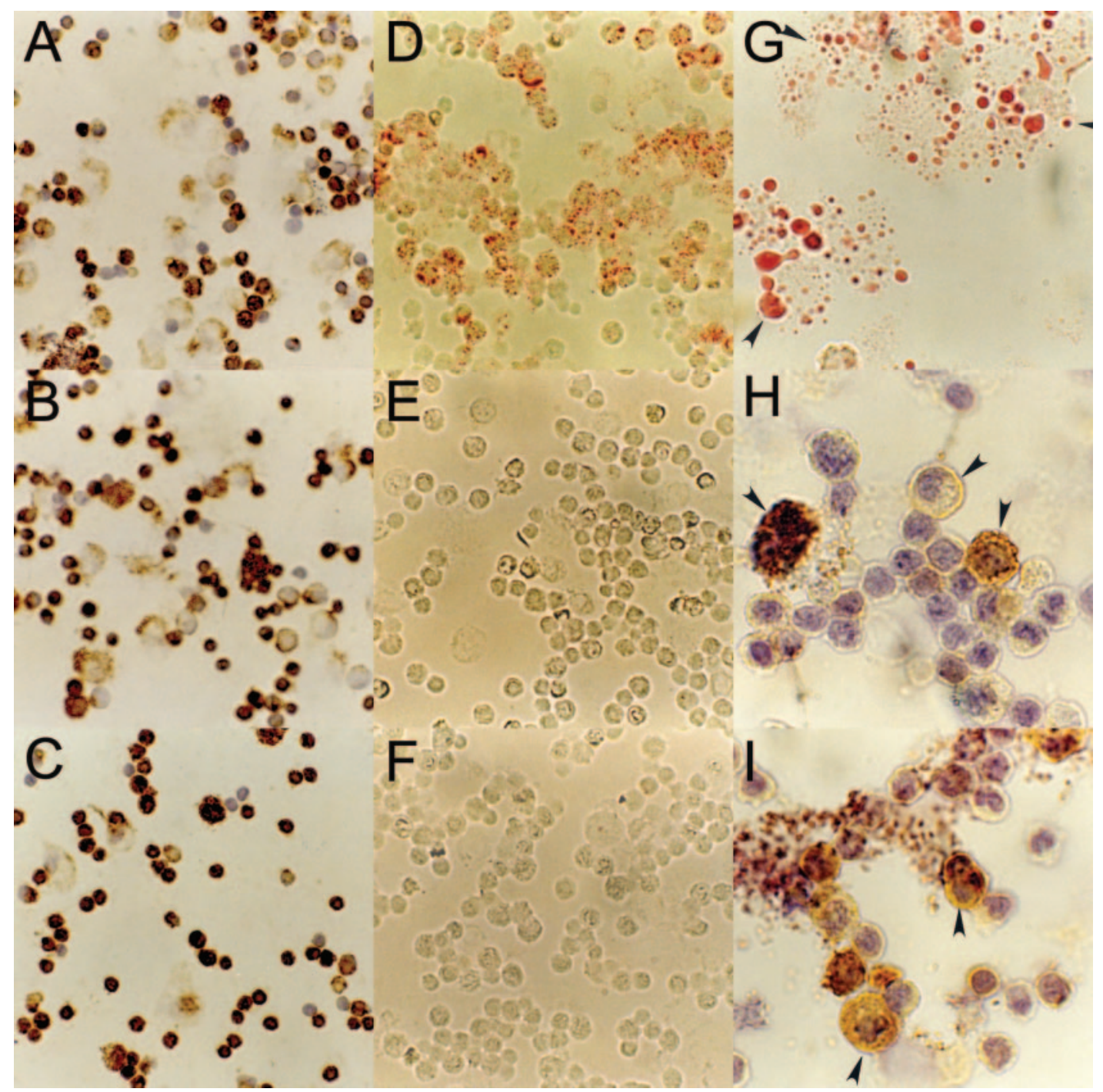

Figure 1. Chlamydia pneumoniae infection induces accelerated macrophage foam cell formation in vivo in recruited peritoneal macrophages. Immunohistochemical staining (brown) with monocyte/macrophage subset antibody (A to C) detects equivalent number in cell smears from Tg53-Cpn rats (A), Tg53-spg mock-infected rats (B), and nontransgenic-Cpn rats (C). Oil-red-O staining detects lipid-laden (stained red) foam cells only in Tg53Cpn rats (D) in contrast to Tg53-spg mock-infected rats (E) and Cpn-infected nontransgenic rats (F). High magnification view reveals macrophage foam cell detail of cell smear from Tg53-Cpn rat (G). Immunohistochemical staining with Cpn anti-HSP60 antibody detects positive staining (brown color) in macrophages of both Tg53-Cpn (H) and nontransgenic-Cpn-infected rats (I). Original magnification: 400x (A to F); 1000x (panels G to I).

Only 5 significant gene expression changes met biological replication standards (21) comparing Tg53-Cpn with either control (Table 2). Three genes are induced in Tg53-Cpn: heat shock protein $70 \mathrm{kD}$ protein 5 (also referred to as glucose-regulated protein 78) or immunoglobulin heavy chain binding protein (Hspa5 or GR78 or BiP, respectively), interferon inducible protein-10 (IP-10), and cytochrome p450-1A1 (see Table 2). Two genes are de-induced in Tg53-Cpn: insulin-like growth factor binding protein 2 (IGF-bp2) and cytochrome p450-PB1 (see Table 2). These gene expression changes were not associated with onset of overt-CAD (19).

\section{Cpn Infection Accelerates Onset of Overt CAD}

Having documented that Cpn infection accelerates macrophage foam cell formation, we next investigated whether this phenomenon would affect CAD progression. We inoculated Tg53 rats intranasally to simulate the human disease scenario. We inoculated at 5 mo of age knowing that at 6 mo, early vulnerable plaque formation begins in the proximal right coronary artery of Tg53 rats on regular rat diet (17). Using a titer previously demonstrated in mouse models not to be lethal $(10)$, we infected Tg53 rats $(n=4)$ and control nontransgenic Dahl S rats $(n=3)$ with $1 \times 10^{7}$ ifu of purified infectious Cpn. No respiratory distress was noted in the immediate $2 \mathrm{wk}$ after intranasal inoculation. As shown in Table 3, survival was decreased in Tg53-Cpn rats compared with nonTg$C p n$ rats $(P=0.009)$. With this small number, it was evident that the $C p n$-infection did not cause decreased survival by itself as reported previously for mice (10). Having attained significant $P$ values with a small group number, this experiment was terminated at $276 \mathrm{~d}$ demonstrating that Cpn does not cause an infection-induced lethality at the inoculum dose given in control nonTg-Cpn rats. 
A.

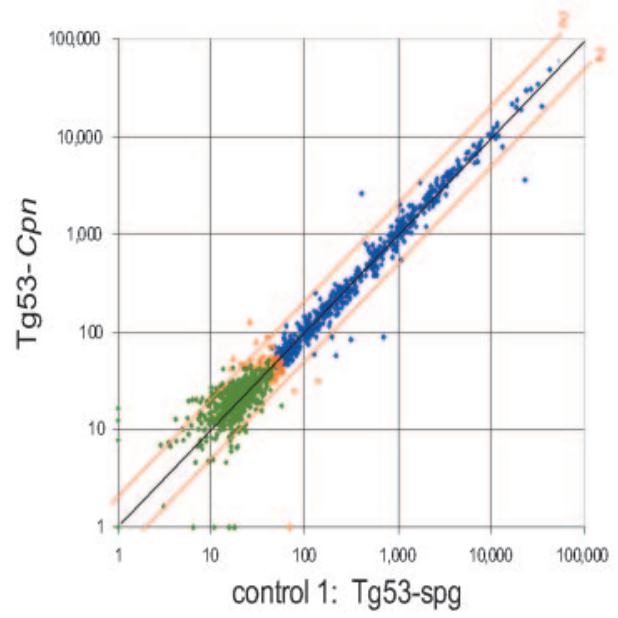

B.

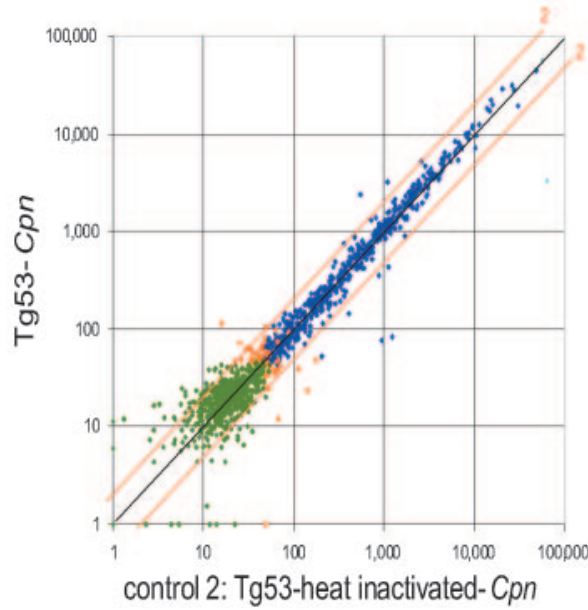

C.

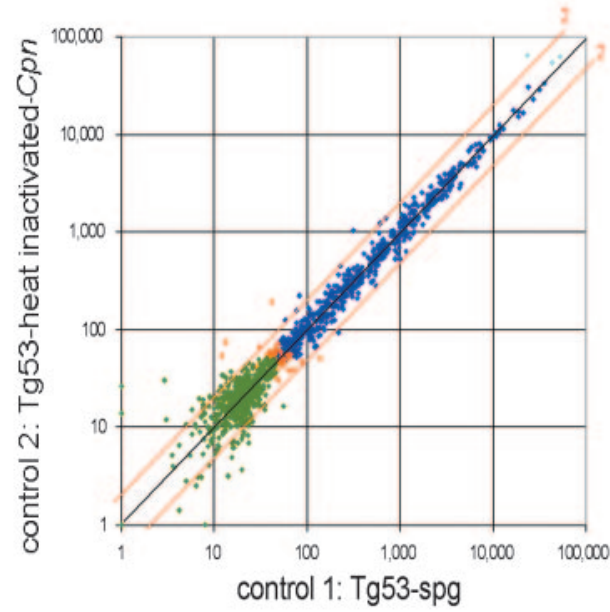

Figure 2. Scatter plots of transcription profiles of recruited peritoneal macrophages comparing Tg53-Cpn, Tg53-spg, and Tg53-Cpn INACTIVATED(NACT). SCatter plot of transcription profiles (1046 unique genes) reveals expression differences between Tg53-Cpn and Tg53-spg (plot A) and between Tg53-Cpn and Tg53-Cpn INACT (plot B) in contrast to minimal differences between Tg53-spg and Tg53-Cpn ${ }^{\text {INACT }}$ (plot C). Data points represent log 10 of normalized signals (signal - local background); threshold = local background; $\bullet$, both values $>$ threshold; $\diamond, 1$ value $<$ threshold; $\bullet$, both values $<$ threshold.; red line denotes 2 -fold change; induction as points above the line, deinduction as points below the line.

To test the hypothesis that decreased survival was specific to Cpn, we inoculated Tg53 rats with a Chlamydial mouse pneumonitis strain (MoPn). As previously reported in LDLr knockout mice, MoPn did not cause any significant morbidity (10). Analysis of life span comparing Tg53-Cpn rats $(n=4)$ with Tg53-MoPn rats $(n=5)$ was significant ( $t$ test, $P=0.038)$. Survival analysis comparisons showed that Tg53-Cpn rats with combined controls further increased significance of differences (log rank sum test, $P=0.006)$. Collectively, these data show that C $p n$-infection causes decreased survival in hyperlipidemic Tg53 rats when inoculated at a time point associated with early vulnerable plaque development in the model (17).
In order to determine that decreased survival in Tg53-Cpn rats is associated with end-stage coronary artery disease, cardiac histology was done to assess coronary lesions. Histological analysis of end-stage Tg53-Cpn Masson-trichrome stained sections detect myocardial infarction (Figure 3A), AHA Type VI "culprit" coronary lesions (see Figure 3B through $3 \mathrm{H}$ ) with luminal (see Figure $3 \mathrm{~B}$ and $3 \mathrm{~F}$ ) and intraplaque thrombi (see Figure $3 \mathrm{C}$ and $3 \mathrm{E}$ ), intraplaque hemorrhages (see Figure 3B to $3 \mathrm{C}$ and $3 \mathrm{E}$ ), and endothelial erosion (see Figure 3E and 3G). PTAH-stained fibrin is detected within the lesion (see Figure 3D) and in the luminal thrombus (see Figure 3H). Platelets are detected in the thrombus (see Figure $3 \mathrm{~F}$ and $3 \mathrm{H}$ ). These histological features provide compelling evidence that decreased

Table 2. 1046-Gene microarray transcription profile analysis ${ }^{a}$

Fold-change in gene expression in recruited peritoneal macrophages from Tg53 rats

\begin{tabular}{|c|c|c|c|c|c|c|}
\hline \multirow{2}{*}{ Unigene ID nr } & \multicolumn{3}{|c|}{ Expression units } & \multicolumn{2}{|c|}{ Fold-change } & \\
\hline & Cpn(3) & $\operatorname{spg}(2)$ & iCpn (3) & Cpn/spg & Cpn/iCpn & Functional category \\
\hline
\end{tabular}

Induced

\begin{tabular}{|c|c|c|c|c|c|c|c|}
\hline Hspa5/GRP78/BiP & 11088 & 3191 & 1746 & 1091 & $2 \uparrow$ & $3 \uparrow$ & Stress response \\
\hline IP-10 & 10584 & 2402 & 412 & 550 & $6 \uparrow$ & $4 \uparrow$ & Chemokine \\
\hline $\begin{array}{l}\text { Cytochrome P450-1A1 } \\
\text { einduced }\end{array}$ & 10352 & 1181 & 30 & 36 & $>10 \uparrow$ & $>10 \uparrow$ & Cytochrome P450 system \\
\hline IGF-binding protein 2 & 6813 & 3316 & 63965 & 23231 & $6 \downarrow$ & $>10 \downarrow$ & Growth factor \\
\hline $\begin{array}{l}\text { Cytochrome P450-PB } 1 \\
\text { eference }\end{array}$ & 7245 & 76 & 316 & 949 & $4 \downarrow$ & $>10 \downarrow$ & Cytochrome P450 system \\
\hline G3-PDH & 54911 & 6234 & 6113 & 5668 & nc & nc & RNA level standard control \\
\hline
\end{tabular}

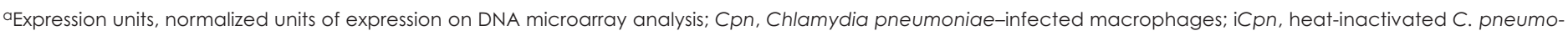

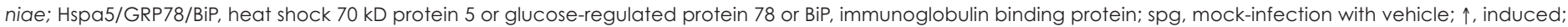
$\downarrow$, de-induced; IP-10, interferon $\gamma$-inducible protein-10; G3-PDH, glyceraldehyde-3-phosphate dehydrogenase; nc, no change in expression. 


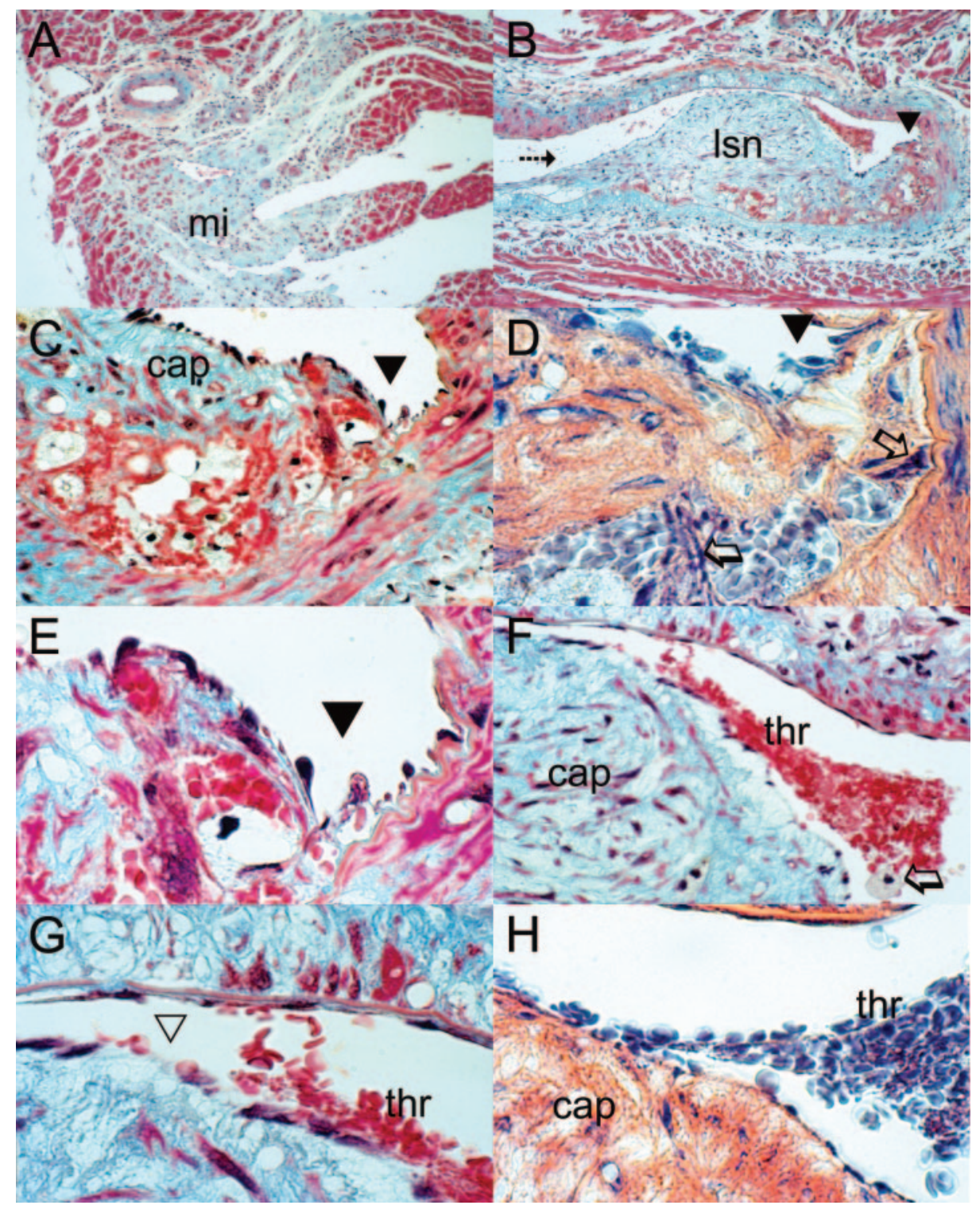

Figure 3. Histological analysis of cardiac pathology in Tg53-Cpn rat hearts at onset of empirical distress identified severe CAD as most likely cause of decreased survival. A: Masson-trichrome (MT)-stained section reveals myocardial infarction (mi) area in Tg53-Cpn right ventricle with variable stages of fibrosis (stained light blue) and loss of myocardiocytes (stained red). B: MT-stained section of Tg53-Cpn proximal right coronary artery lesion reveals severely occlusive lesion (Isn) with attached thrombi and downstream lesion shoulder erosion ( $\mathbf{\nabla}$ ). Vessel remodeling is evident, as well as paucity of smooth muscle cells (stained pink in the media) in remodeled vascular section. The lesion cap is relatively thin at the downstream shoulder ( $\rightarrow$, direction of blood flow) compared with the central bulk of Isn. The lesion shoulder exhibits vulnerable plaque features such as foam cell enrichment, intraplaque hemorrhage, thrombi, and extracellular lipid accumulations. Original magnification 200x. C: High magnification (original magnification 400x) of MT-stained lesion shoulder ( $\mathbf{\nabla}$ ) reveals thin cap (cap) and greater area of foam cells, intraplaque hemorrhage, thrombi, and extracellular lipid. D: PTAH stained adjacent section of lesion shoulder $(\mathbf{\nabla})$ reveals PTAH-stained fibrin $(\Rightarrow)$ in the intimal lesion surrounded by paler stained red blood cells. (Magnification 400x). E: Higher magnification (original magnification 1000x) of MT-stained lesion shoulder ( $\mathbf{\nabla}$ ) reveals endothelial erosion, intraplaque hemorrhage (red blood cells stained red), and extracellular lipid accumulations within the lesion. F: High magnification (400x) of luminal thrombus (thr) reveals attachment over thick-capped (cap) area of lesion with endothelial erosion-seen better in $G$ under higher magnification (1000x). Red blood cells and platelets evident. $\nabla$, site of endothelial erosion. H: PTAH-stained section shows thrombus (thr) attached to area of endothelial erosion and reveals platelets within thrombus.

survival in Tg53-Cpn rats is due to CAD destabilization. We next determined whether abundant expression of matrix metalloproteinases would be detected as reported for human coronary plaques associated with Cpn-infection (23). Immunohistochemical analysis of an adjacent serial section reveals abundant matrix met- alloproteinase 3 expression in Tg53-Cpn "culprit" coronary lesion (Figure 4A). Additionally, left atrial thrombi were detected in $2 / 2$ Tg53-Cpn rat hearts analyzed (see Figure 4B through 4C). Left atrial thrombi have not been previously detected in any Tg53 rat heart previously analyzed (17, unpublished data). Histological analysis 
Table 3. Survival analysis

\begin{tabular}{lccc} 
Study groups & $\begin{array}{c}\text { Tg53-Cpn } \\
(n=4)\end{array}$ & $\begin{array}{c}\text { Tg53-MoPn } \\
(n=5)\end{array}$ & $\begin{array}{c}\text { NonTg-Cpn } \\
(n=3)\end{array}$ \\
\hline Mean survival (d $\left.{ }^{b}\right)$ & $200.3 \pm 18$ & $240.8 \pm 10$ & $274.0 \pm 2$ \\
$t$ test (compared & & $P=0.038$ & $P=0.009$ \\
with Tg53-Cpn) & & &
\end{tabular}

aCpn, inoculated with Chlamydia pneumoniae; MoPn, inoculated with Chlamydial mouse pneumonitis; NonTg, non-transgenic Dahl S rats.

bexperiment stopped at 276 d because lifespan differences already attained significant $P$ value with small group number; new control Tg53-MoPn then added.

of left atrial thrombi detects organization, leukocyte infiltration and layering (see Figure 4B and 4C)-most likely reflecting multiple thrombotic events. PTAH staining confirms presence of fibrin in these thrombi (data not shown).

\section{DISCUSSION}

\section{Modeling the Impact of $C p n$ Infection on CAD Progression}

The data provide evidence that a one-time, untreated nasopharyngeal-repiratory infection with Cpn can enhance progression to overt-CAD in hyperlipidemic Tg53 Dahl S rats. This Cpn-CAD interaction is hyperlipidemia-dependent, since Cpn-infected nontransgenic Dahl S rats did not exhibit CAD (data not shown) nor decreased survival. Our data are concordant with observations on the impact of Cpn on aortic atherosclerosis in different mouse and rabbit models $(10,11,13)$. The successful modeling of Cpn effects on CAD progression demonstrates that Cpn infection is a causal risk factor rather than just a bystander. The data also indicate that
Cpn infection is not a necessary nor sufficient etiological factor for coronary atherosclerosis, consistent with a risk factor paradigm (24). The detection of left atrial thrombi in Tg53-Cpn raises the question that it might comprise another Cpn-atherosclerosis interaction paradigm deserving more study.

\section{Accelerated Macrophage Foam Cell Formation}

Parallel to observations of human monocytes in cell culture (20), in vivo recruited peritoneal macrophages infected once with Cpn exhibit a temporal and quantitative enhancement of foam cell formation. Given the context that human unstable plaques in acute coronary syndromes are foam-cell rich, especially in areas of plaque rupture - an observation recapitulated in overt-CAD Tg53 rats - it could be expected that Cpn-induced acceleration of foam cell formation increases risk of coronary plaque destabilization or progression to overt-CAD. Although other mechanisms are not ruled out or in, accelerated foam cell formation presents a valid cell-based hypothesis for Cpninduced overt-CAD progression as suggested by in vitro studies wherein $C p n$-infection also accelerates macrophage foam cell formation in cultured human monocytes exposed to oxidized LDL (20).

\section{Insights From Transcription Profile of $C p n$-Induced Accelerated Macrophage Foam Cells}

Although limited to 1046 unique gene microarray and to the experimental design used here, the distinct transcription profile of macrophages exposed to infectious Cpn compared with macrophages exposed to inactivated-Cpn or vehicle alone is significant (ANOVA $P<0.001$ ). These data parallel the histological profile of increased macrophage foam cells that distinguishes

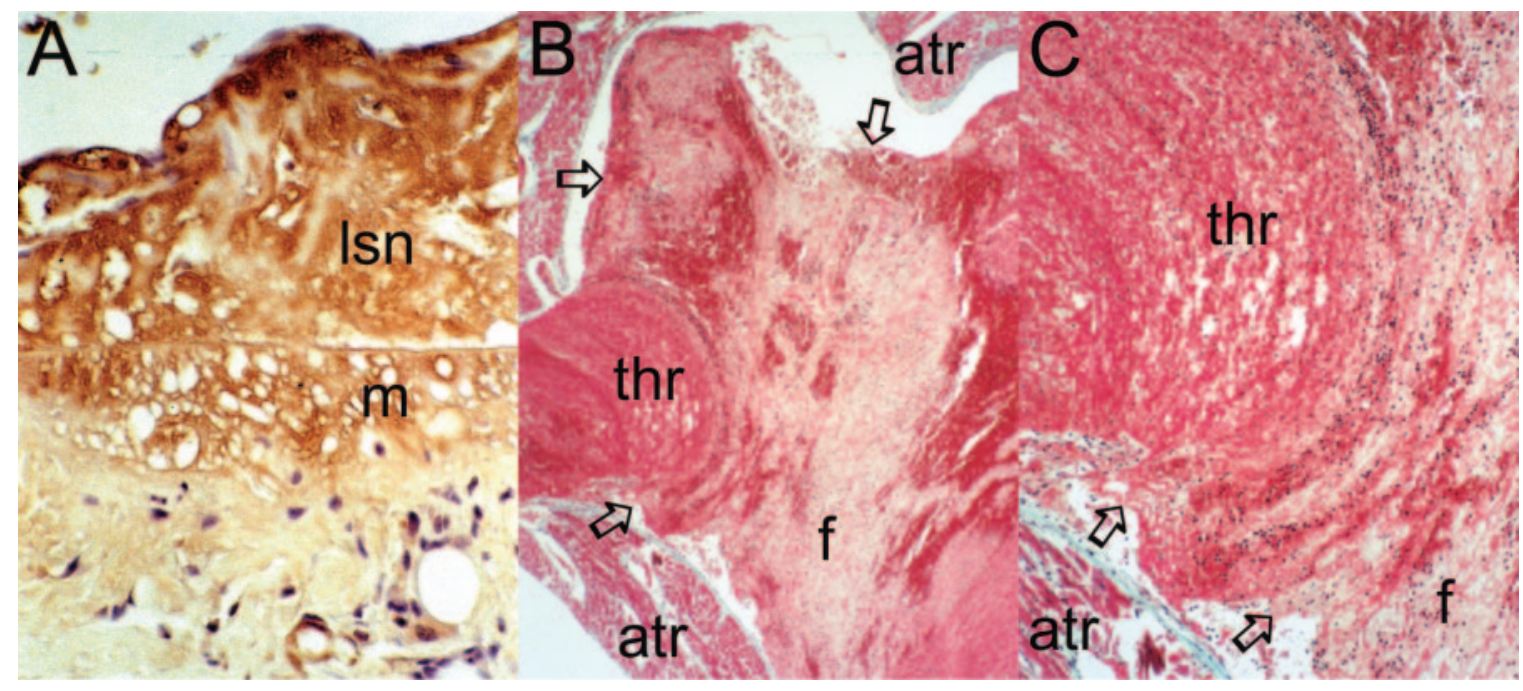

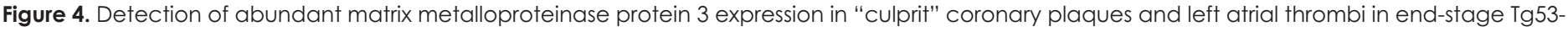

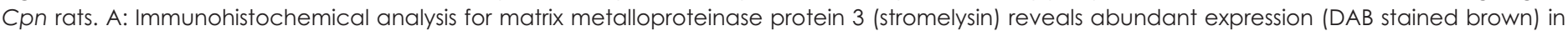

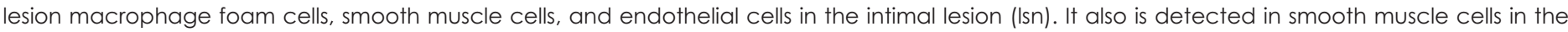

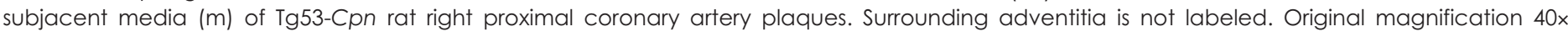

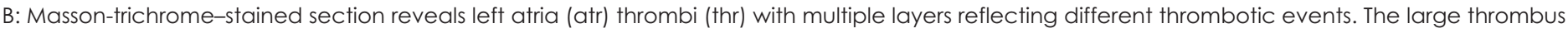

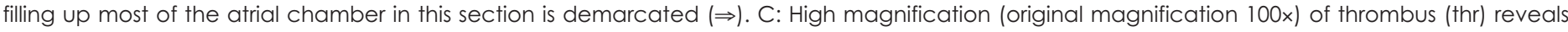

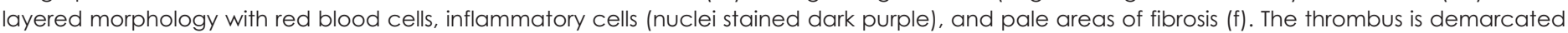
$(\Rightarrow)$ from the left atrial wall (atr). 
peritoneal macrophages exposed to infectious Cpn. Interestingly, only 5 genes exhibit expression changes confirmed in both experimental designs, Hspa5/GR78/BiP, IP-10, IGF-bp2, cytochrome P450-1A1, and cytochrome p450-PB1, in contrast to many more expressed genes that remain unchanged including different interleukins and receptors, TNF ligands and receptors, caspase 3 and 6 , and cell cycle regulators. This brings confidence to the specificity of detected transcription profile changes. Correlation with known functions reveals that 3 of 5 genes might play a role in Cpn-CAD interaction. Induction of IP-10 stimulates T-cell adhesion to endothelial cells and modulates T-cell, monocyte, and neutrophil chemoattraction $(25,26)$ cell types, which have been implicated in vulnerable plaque destabilization $(1,17)$. Induction of Hspa5/ GR78/BiP, an endoplasmic reticulum stress response protein, is thought to contribute to atherosclerosis risk in hyperhomocysteinemia (27). As a negative effector of cell survival (28), decreased IGF-bp2 would favor macrophage foam cell survival allowing for its many plaque progression roles. On the other hand, decreased expression of cytochrome P450-PB1 and induction of cytochrome P450-1A1 most likely represent responses to infectious agents $(29,30)$.

In summary, the data provide evidence that Cpn is a causal risk factor contributing to CAD progression in the Tg53 rat model. The detection of accelerated macrophage foam cells marked by a distinct transcription profile provides a framework for further study.

\section{ACKNOWLEDGMENTS}

We acknowledge the AHA-grant-in-aid, Evans intersection collaborative award, and NIH grant RO1 HL62857. We thank Dr Guongming Zhong (University of Texas Health Center at San Antonio) for providing the Cpn strain AR-39. We thank Dr Peter A Rice for support and helpful discussions.

Address correspondence and reprint requests to Victoria LM Herrera, Whitaker Cardiovascular Institute, Boston University School of Medicine, 700 Albany Street, W-609, Boston MA 02118. Phone: 617638-4020; fax: 617-638-4066; e-mail: vherrera@bu.edu.

Submitted April 9, 2003; accepted for publication April 28, 2003.

\section{REFERENCES}

1. Delager-Pedersen S, Pederson EM, Ringgaard S, Falk E. (1999) Coronary artery disease: plaque vulnerability, disruption and thrombosis. In: The vulnerable atherosclerotic plaque. Fuster V (ed.) Futura, Inc., New York, N.Y. pp. 1-23.

2. Saikku $P$ et al. (1988) Serological evidence of an association of a novel Chlamydia, TWAR, with chronic coronary heart disease and acute myocardial infarction. Lancet 2:983-6.

3. Puolakkainen M, Kuo CC, Shor A, Wang SP, Grayston JT, Campbell LA (1993) Serological response to Chlamydia pneumoniae in adults with coronary arterial fatty streak and fibrolipid plaques. J. Clin. Microbiol. 31:2212-4.

4. Ong G, Thomas BJ, Mansfield AO, Davidson BR, Taylor-Robinson D. (1996)
Detection and widespread distribution of Chlamydia pneumoniae in the vascular system and its possible implications. J. Clin. Pathol. 49:102-6.

5. Ramirez JA. (1996) Isolation of Chlamydia pneumoniae from the coronary artery of a patient with coronary atherosclerosis. The Chlamydia pneumoniae/Atherosclerosis Study Group. Ann. Intern. Med. 125:979-82.

6. Gurfinkel E, Bozovich G, Beck E, Testa E, Livellara B, Mautner B, (1999) Treatment with the antibiotic roxithromycin in patients with acute non-Q-wave coronary syndromes: the final report of the ROXIS Study. Eur. Heart J. 20:121-7.

7. Anderson JL et al. (1999) Randomized secondary prevention trial of azithromycin inpatients with coronary artery disease and serological evidence for Chlamydia pneumoniae infection. Circulation 99:1540-7.

8. Muhlstein JB, Anderson JL, Hammon EH, Zhao L, Trehan S, Schowobe E, Carlquis JF. (1998) Infection with Chlamydia pneumoniae accelerates the development of atherosclerosis and treatment with azithromycin prevents it in a rabbit model. Circulation 97:633-6.

9. Blessing E, Campbell LA, Rosenfeld ME, Chough N, Kuo CC. (2001) Chlamydia pneumoniae infection accelerates hyperlipidemia induced atherosclerotic lesion development in C57BL/6J mice. Atherosclerosis 158:13-7.

10. Hu H, Pierce GN, Zhong G. (1999) The atherogenic effects of Chlamydia are dependent on serum cholesterol and specific to Chlamydia pneumoniae. J.Clin. Invest. 103:747-53.

11. Moazed T, Campbell LA, Rosenfeld ME, Grayston JT, Kuo CC. (1999) Chlamydia pneumoniae infection accelerates the progression of atherosclerosis in apolipoprotein E-deficient mice. J. Infect. Dis. 180:238-41.

12. Burnett MS, Gaydos CA, Madico GE, Glad SM, Paigen B, Quinn TC, Epstein SE. (2001) Atherosclerosis in apoE knockout mice infected with multiple pathogens. J. Infect. Dis. 183:226-31

13. Ezzahiri R et al. (2002) Chlamydophila pneumoniae (Chlamydia pneumonaie) accelerates the formation of complex atherosclerotic lesions in Apo E3-Leiden mice. Cardiovasc. Res. 56:269-76.

14. Caligiuri G, Rottenberg M, Nicoletti A, Wigzell H, Hansson GK. (2001). Chlamydia pneumoniae infection does not induce or modify atherosclerosis in mice. Circulation 103:2834-8.

15. Aalto-Setala K, Laitinen $K$, Kerkkila L, Leinonen $M$, Jauhiainen $M$, Ehnholm $C$ Tamminen M, Puolakkainen M, Penttila I, Saikku P. (2001). Chlamydia pneumoniae does not increase atherosclerosis in the aortic root of apolipoprotein Edeficient mice. Arterioscler. Thromb. Vasc. Biol. 21:578-84.

16. Blessing E, Nagano S, Campbell LA, Rosenfeld ME, Kuo CC. (2000) Effect of Chlamydia trachomatis infection on atherosclerosis in apolipoprotein E-deficient mice. Infect. Immun. 68:7195-7.

17. Herrera VLM et al. (2001) Hypertension exacerbates coronary artery disease in transgenic hyperlipidemic Dahl salt-sensitive hypertensive rats. Mol. Med. 7:831-44

18. Kalayoglu MV, Byrne GI. (1998) A Chlamydia pneumoniae component that induces macrophage foam cell formation is chlamydial lipopolysaccharide. Infect. Immunity 66:5067-72.

19. Herrera VLM, Didishvili T, Lopez LV, Ruiz-Opazo N. (2002) Differential regulation of functional gene clusters in overt coronary artery disease in a transgenic atherosclerosis-hypertensive rat model. Mol. Med. 8:367-75.

20. Kalayoglu MV, Byrne Gl. (1998) Induction of macrophage foam cell formation by Chlamydia pneumoniae. J. Infect. Dis. 177:725-9.

21. Churchill GA. (2002) Fundamentals of experimental design for CDNA microarrays. Nature Genetics (Suppl) 32:490-5.

22. Quackenbush J. (2002) Microarray data normalization and transformation. Nature Genetics (Suppl) 32:496-501.

23. Kol A, Sukhova GK, Lichtman AH, Libby P. (1998) Chlamydial heat shock protein 60 localizes in human atheroma and regulates macrophage tumor necrosis factor-alpha and matrix metalloproteinase expression. Circulation 98:300-7.

24. Epstein SE, Zhou YF, Zhu J. (1999). Infection and atherosclerosis: emerging mechanistic paradigms. Circulation 100:e20-8.

25. Taub D, Sayers T, Carter C, Ortaldo J. (1995) Alpha and beta chemokines induce NK cell migration and enhance NK-mediated cytolysis. J. Immunol. 155:3877-88.

26. Neville LF, Mathiak G, Bagasra O. (1997) The immunobiology of interferon- $\gamma$ inducible protein $10 \mathrm{kD}$ (IP-10): a novel, pleiotropic member of the C-X-C chemokine superfamily. Cytokine Growth Factor Rev. 8:207-19.

27. Kokame K, Kato H, Miyata T. (1998). Nonradioactive differential display cloning of genes induced by homocysteine in vascular endothelial cells. Methods 16:434-43.

28. Kuhl NM, Keyser J, De Vries H, Hoekstra D. (2002) Insulin-like growth factor binding proteins-1 and -2 differentially inhibit rat oligodendrocyte precursor cell survival and differentiation in vitro. J. Neurosci. 69:207-16.

29. Coombs GH, Wolf CR, Morrison VM, Craft JA. (1990) Changes in hepatic xenobiotic-metabolising enzymes in mouse liver following infection with Leishmania donovani. Mol. Biochem. Parasitol. 41:17-24.

30. Sewer MB, Koop DR, Morgan ET. (1996) Endotoxemia in rats is associated with induction of the P450A subfamily and suppression of several other forms of cytochrome P450. Drug Metabolism Dispos. 24:401-7. 